\title{
Bortezomib in mantle cell lymphoma: comparative therapeutic outcomes
}

\author{
This article was published in the following Dove Press journal: \\ Therapeutics and Clinical Risk Management \\ 6 November 2015 \\ Number of times this article has been viewed
}

\section{Nishanth Vallumsetla \\ Jonas Paludo \\ Prashant Kapoor}

Division of Hematology, Department of Internal Medicine, Mayo Clinic, Rochester, MN, USA
Correspondence: Prashant Kapoor Division of Hematology, Department of Internal Medicine, Mayo Clinic, 200 First Street SW, Rochester, MN 55905, USA $\mathrm{Tel}+\mid 507538059$ I

Fax + I 5075380590

Email kapoor.prashant@mayo.edu

\begin{abstract}
Mantle cell lymphoma (MCL) is an incurable, typically aggressive subtype of non-Hodgkin lymphoma, accounting for $4 \%-7 \%$ of newly diagnosed non-Hodgkin lymphoma cases. Chemoresistance commonly ensues in MCL, and patients with this heterogeneous disease invariably relapse, underscoring the unmet need for better therapies. Over the past few years, several novel agents with promising activity and unique mechanisms of action have been deemed effective in MCL. Bortezomib is a reversible proteasome inhibitor, approved as a single agent for patients with relapsed/refractory MCL who have received at least one prior line of therapy. Addition of bortezomib to chemoimmunotherapies has demonstrated good tolerability and superior efficacy, both in the upfront and salvage settings, and recently one such combination of bortezomib plus rituximab, cyclophosphamide, doxorubicin, and prednisone was approved as a frontline regimen in untreated patients with MCL. This review examines the role of bortezomib in a multitude of clinical settings and ongoing clinical trials designed to optimize its integration in the current treatment paradigms of MCL.
\end{abstract}

Keywords: non-Hodgkin lymphoma, proteosome inhibitor, treatment

\section{Introduction}

Mantle cell lymphoma (MCL) is a strikingly male predominant, distinct subtype of non-Hodgkin lymphoma (NHL), accounting for 4\%-7\% of all newly diagnosed adult NHL in the USA and Europe. Approximately 5,000 cases are diagnosed annually in the USA, with the median age being approximately 68 years at diagnosis. ${ }^{1-3}$ An increasing incidence with age and an overall age-adjusted incidence rate of 0.64 per 100,000 person years has been reported for MCL. ${ }^{3} \mathrm{MCL}$ involves mature B-cells that are characterized by translocation $\mathrm{t}(11 ; 14)(\mathrm{q} 13 ; \mathrm{q} 32)$, resulting in overexpression of cyclin D1. Immunophenotyping in MCL typically reveals a CD5+, CD20+, CD10-, FMC7+, CD23-, CD43+, and cyclin D1+ profile. Although lymph node enlargement is the primary presenting feature in the majority of the cases, a number of patients may additionally demonstrate extranodal disease, including involvement of the bone marrow, peripheral blood, gastrointestinal tract, central nervous system, or liver. ${ }^{4}$

\section{Management-related challenges}

MCL remains an incurable malignancy and usually has an aggressive disease course, with a majority of patients requiring initiation of treatment upon diagnosis itself. However, the outcomes of patients with this biologically heterogeneous disease are disparate, and management-related challenges are formidable. A small proportion $(10 \%-15 \%)$ of patients generally presents with splenomegaly, marrow and peripheral blood involvement, normal serum lactate dehydrogenase in the absence of lymphadenopathy and exhibits an indolent disease course. A retrospective study has suggested 
that a "wait and watch" approach does not necessarily affect the overall survival (OS) adversely in such patients. ${ }^{5}$ However, the means to accurately identify this subset still remains unclear. Recent studies have found that although non-nodal indolent MCL and conventional MCL largely share a genetic profile, the expression of SOX11 gene was the most notable of the differences seen. ${ }^{6}$ In an independent case series of 112 patients with MCL, the lack of SOX11 protein expression correlated with a better prognosis. Highly mutated immunoglobulin heavy chain variable gene is another independent factor that has been found to be associated with indolent MCL. ${ }^{7} \mathrm{Ki}-67$ index, a measure of tumor proliferative activity, along with MCL International Prognostic Index (MIPI) score may be useful tools but have not yet been validated as indicators for initiation of therapy. ${ }^{8}$

No standard frontline therapeutic approach currently exists for patients with an aggressive disease. Most, even the autologous stem-cell transplant (ASCT) eligible and young patients, eventually relapse. A median survival of only up to 5 years is demonstrable even in patients with the lowest MIPI score. ${ }^{9}$

\section{Outline of current therapies}

The therapeutic strategies for MCL are based primarily on the disease stage, age, and the fitness of the patients. MIPI is used to classify patients on the basis of age, performance status, lactate dehydrogenase, and leukocyte count into three subgroups: low, intermediate, and high risk. ${ }^{9}$ This prognostic index currently serves as an important tool facilitating risk-adapted treatment decisions in advanced-stage patients. Those requiring treatment at diagnosis are broadly classified as 1 ) young (typically $<65$ years) and/or fit individuals who can tolerate intensive regimens, usually involving cytarabinebased induction followed by conditioning (generally, BEAM, carmustine, etoposide, cytarabine, melphalan) with stem-cell rescue, 2) older individuals with good performance status who may not be ASCT eligible but can tolerate relatively intense regimens and continuous/maintenance therapy, and 3) frail patients who are suitable candidates for only milder chemoimmunotherapies.

Currently, rituximab, a monoclonal chimeric antibody targeting the CD20 antigen expressed on B-cells, is the most commonly used agent in MCL that can easily be combined with chemotherapeutic regimens. A meta-analysis of three trials, involving 260 patients, has shown rituximab plus chemotherapy (R-chemo) to be associated with superior outcomes compared with chemotherapy alone. ${ }^{10}$ In a Surveillance, Epidemiology, and End Results (SEER)-Medicare database study of older patients with MCL, R-chemo has shown to improve OS (median 37 months) in comparison with chemotherapy alone (median 27 months), along with a lower all-cause and cancer-specific mortality in the frontline setting. ${ }^{11}$ Therefore, rituximab, with its tolerability and probable survival benefit, is currently routinely used in most patients with MCL.

Common combination regimens include R-Hyper CVAD (rituximab + cyclophosphamide, doxorubicin, vincristine, dexamethasone alternating with methotrexate and cytarabine), R-CHOP (rituximab, cyclophosphamide, doxorubicin, vincristine, and prednisone), the Nordic regimen of maxi-CHOP alternating with rituximab and high-dose cytarabine, R-CVP (rituximab, cyclophosphamide, vincristine, and prednisone), R-FC (rituximab, fludarabine, and cyclophosphamide), BR (bendamustine with rituximab), R-CHOP alternating with R-DHAP (rituximab, dexamethasone, cytarabine, and cisplatin), VcR-CVAD (bortezomib, rituximab, cyclophosphamide, vincristine, doxorubicin, and dexamethasone), and the more recently studied VR-CAP (bortezomib, rituximab, cyclophosphamide, doxorubicin, and prednisone). Consolidation with ASCT is considered in patients achieving at least a partial response (PR) with induction. Addition of rituximab and ASCT to chemotherapeutic regimens has been shown to independently improve durability of response in patients with MCL. ${ }^{12}$ Rituximab maintenance is a viable effective alternative to ASCT in older ASCT-ineligible patients. ${ }^{13}$

In the younger and/or fit patients, R-CHOP followed by ASCT consolidation, results in a superior overall response rate (ORR; 94\% vs 75\%; $P=0.005$ ), complete response (CR) rate $(34 \%$ vs $7 \% ; P<0.001)$, and time-to-treatment failure (TTF) compared with CHOP alone (median 21 vs 14 months; $P=0.013) .{ }^{14}$ More recently, in a large randomized Phase III trial involving 455 patients, R-CHOP alternating with R-DHAP followed by high-dose cytarabine-based myeloablative regimen with stem-cell rescue was shown to result in superior OS (median not reached (NR) vs 82 months; $P=0.04$ ) in comparison with R-CHOP followed by ASCT, without any significant difference in toxicities associated with the two approaches. ${ }^{15}$ There are currently no standard therapies for MCL, and the patients' age, comorbidities, and performance status need to be taken into account before recommending an induction regimen (Table 1).

In patients who are fit enough to tolerate intense chemoimmunotherapy, R-Hyper CVAD alternating with rituximab plus high-dose methotrexate/cytarabine regimen has been proposed as an alternative therapeutic option. Exceptionally high CR rate of $87 \%$ and an 8 -year OS of $56 \%$ have 


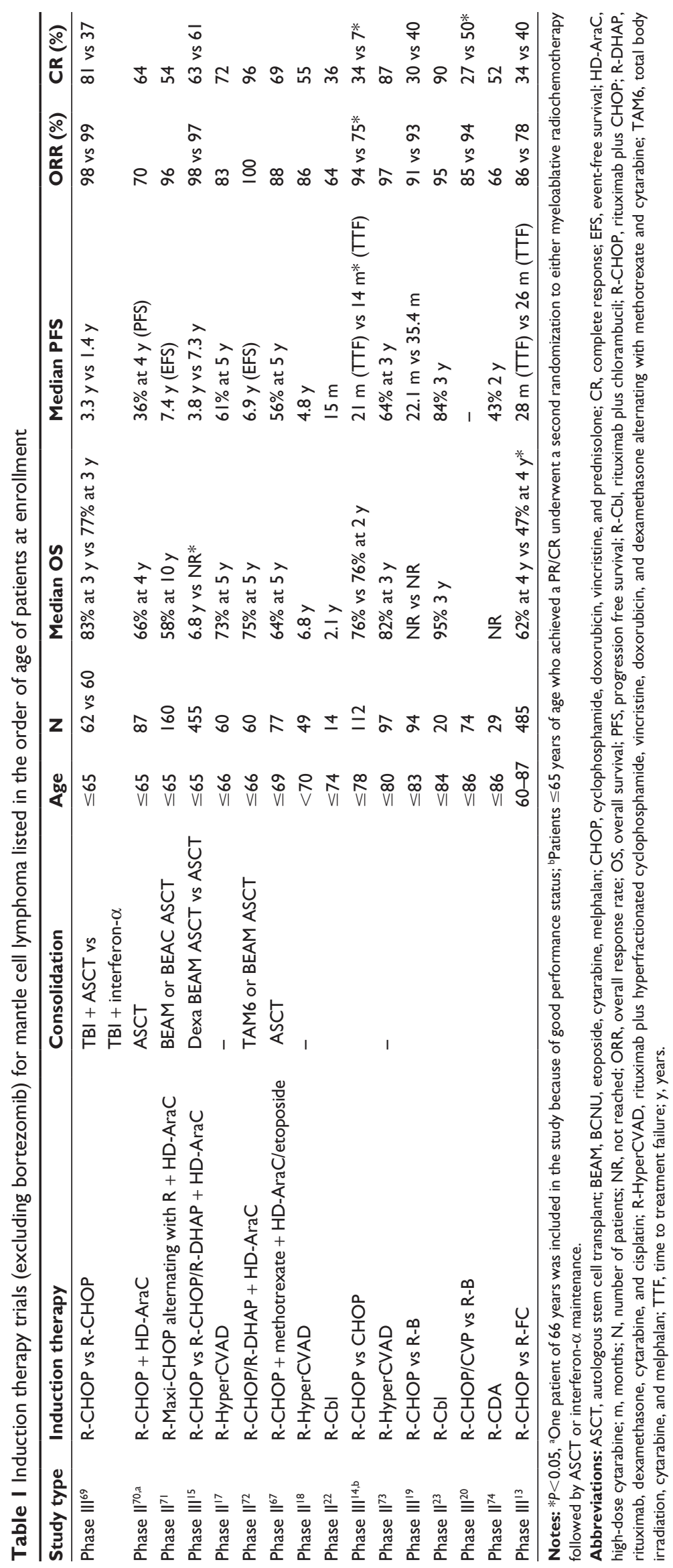


been reported with this approach in a Phase II single-center trial, but similar results could not be replicated in two other Phase II multicenter studies. ${ }^{16-18}$ A high dropout rate, especially among patients older than 65 years, irrespective of the comorbidities, was observed in these trials due to therapyrelated toxicities, and as such, precludes use of this regimen in older patients.

A randomized trial involving 485 patients with MCL, $\geq 60$ years of age or older, comparing R-CHOP and R-FC demonstrated a significant survival benefit with R-CHOP (4-year OS, 62\% vs 47\% with R-FC; $P=0.005$; Table 1). ${ }^{13}$ Addition of maintenance rituximab (MR) in the patients responding to R-CHOP in this study also showed improved survival (4-year OS, $87 \%$ with MR vs $63 \%$ with interferon alfa maintenance; $P=0.005)$.

Bendamustine rituximab (BR) is another suitable option with a better toxicity profile and longer progression-free survival (PFS) than R-CHOP (PFS, median 35.4 months vs 22.1 months with R-CHOP) and deeper response rates than R-CHOP/R-CVP (CR rate 50\% vs 27\% with R-CHOP/ $\mathrm{R}-\mathrm{CVP}$; CR rate ratio 1.95 in favor of $\mathrm{BR} ; P=0.018$ ) as suggested by two (StiL and BRIGHT) Phase III trials, although no survival advantage has been observed with BR, thus far (Table 1). ${ }^{19,20}$ The impact of MR was not studied in either of these trials. BR regimen has become a preferred induction regimen, and many experts use it outside of clinical trials prior to either consolidation with ASCT in the transplant eligible patients or prior to maintenance with rituximab in the older, fit but transplant ineligible patients with MCL.

Bortezomib is the first of the three novel agents approved in the USA for the treatment of relapsed/refractory MCL. In the European Union, it is approved in combination with rituximab, cyclophosphamide, doxorubicin and prednisone for the treatment of previously untreated patients with MCL who are not candidates for ASCT. It is also the first novel agent to be integrated into the traditional frontline approaches. A number of bortezomib-containing regimens such as VR-CAP, modified R-HyperCVAD \pm bortezomib with MR and RiPAD $+\mathrm{C}$ (rituximab, bortezomib, doxorubicin, dexamethasone, and chlorambucil), discussed in detail in the subsequent sections, have shown promising activity in recent years. In a large randomized Phase III trial, VR-CAP was associated with superior PFS and CR rates compared with R-CHOP, and has recently been approved in the frontline setting. ${ }^{21}$ Less intensive and more tolerable chemoimmunotherapy regimens like BR, chlorambucil plus rituximab (ORR of 64\%-95\%), and PEP-C (prednisone, etoposide, procarbazine, and cyclophosphamide; ORR of $82 \%$ ) are options for the frail/elderly patients. ${ }^{19,22-24}$ Maintaining the quality-of-life (QoL) with symptom palliation is the primary management goal in this challenging subset of patients. Similar to the frontline approaches, there are no recommended sequences of salvage therapies for the patients with relapsed/refractory MCL.

\section{Emerging therapies}

The roles of newer monoclonal anti-CD20 antibodies, obinutuzumab and ofatumumab as alternatives to rituximab, are currently being examined in MCL. ${ }^{25,26}$ Ibrutinib and lenalidomide are other novel agents that have been approved in addition to bortezomib as salvage therapies from different classes for MCL in the USA. In addition to these agents, temsirolimus, an mTOR inhibitor, has been approved in Europe.

Ibrutinib, an irreversible, oral Bruton tyrosine kinase inhibitor, is the most promising of these agents as monotherapy (Table 2). ${ }^{27}$ Its toxicity profile is favorable, with common adverse effects being nausea, fatigue, diarrhea, and edema. Ibrutinib has shown promising activity in combination with BR in an early Phase I trial. ${ }^{28}$

The other two agents, lenalidomide and temsirolimus, have moderate activity (Table 2) as monotherapies in the relapsed/refractory setting, and are currently being investigated in combination with established chemotherapeutic regimens. ${ }^{29-31}$ Single-agent lenalidomide has also been compared with single agents other than bortezomib (chlorambucil, cytarabine, fludarabine, gemcitabine, or rituximab) in the recently reported SPRINT study. The patients were

Table 2 Comparison of outcomes with approved monotherapies in $\mathrm{MCL}$

\begin{tabular}{|c|c|c|c|c|c|c|c|c|}
\hline $\begin{array}{l}\text { Study } \\
\text { type }\end{array}$ & Therapy & Dose & $\mathbf{N}$ & $\begin{array}{l}\text { Median OS } \\
\text { (months) }\end{array}$ & $\begin{array}{l}\text { Median PFS } \\
\text { (months) }\end{array}$ & $\begin{array}{l}\text { ORR } \\
\text { (\%) }\end{array}$ & $\begin{array}{l}\text { CR/CRu } \\
\text { (\%) }\end{array}$ & $\begin{array}{l}\text { PR } \\
\text { (\%) }\end{array}$ \\
\hline Phase $1{ }^{29}$ & Bortezomib & $1.3 \mathrm{mg} / \mathrm{m}^{2}$, days $\mathrm{I}, 4,8, \mathrm{II}$, every $2 \mathrm{I}$ days & 155 & 23.5 & 6.5 & 32 & 8 & 24 \\
\hline Phase $\|^{30}$ & Lenalidomide & $25 \mathrm{mg} /$ day $\mathrm{PO}$ on days $\mathrm{I}-2 \mathrm{I}$ of a 28 -day cycle & 134 & 19 & 4 & 28 & 8 & 20 \\
\hline Phase $\|^{27}$ & Ibrutinib & $560 \mathrm{mg} /$ day & $11 \mathrm{I}$ & $\mathrm{NR}, 58 \%$ at $18 \mathrm{~m}$ & 14 & 68 & 21 & 47 \\
\hline \multirow[t]{2}{*}{ Phase $\| I I I^{31}$} & Temsirolimus & I $75 \mathrm{mg}$ weekly for 3 weeks followed by $75 \mathrm{mg}$ weekly & 54 & 13 & 5 & 22 & 2 & 20 \\
\hline & & $175 \mathrm{mg}$ weekly for 3 weeks followed by $25 \mathrm{mg}$ weekly & 54 & 10 & 3 & 6 & 0 & 6 \\
\hline
\end{tabular}

Abbreviations: $\mathrm{CR}$, complete response; $\mathrm{CRu}$, complete response unconfirmed; $\mathrm{m}$, months; $\mathrm{N}$, evaluable patients; $\mathrm{NR}$, not reached; ORR, overall response rate; OS, overall survival; PFS, progression free survival; PO, per oral; PR, partial response. 
randomized to receive either lenalidomide $(n=170)$ or other monotherapies based on choice of investigators $(n=84)$. A longer PFS was observed with lenalidomide (median 8.7 months vs 5.2 months; $P=0.004) .{ }^{32}$ Lenalidomide plus rituximab has shown an ORR of $56.5 \%$ with a CR of $34.8 \%$ in the relapsed/refractory setting. Median PFS and OS of 14.1 months (95\% CI: 8.2-26.7), and 24.6 months (95\% CI: 16.8-33.7), respectively, were observed. ${ }^{33}$

Idelalisib, an oral PI3K delta isoform-selective inhibitor, has shown encouraging results in a Phase I trial as monotherapy with an ORR of $62 \%$ and an acceptable safety profile in relapsed/refractory MCL. ${ }^{34}$ Phase II studies are further investigating its activity in MCL.

\section{Pharmacology and mode of action of bortezomib}

Bortezomib, a first-in-class proteasome inhibitor, is approved by the US Food and Drug Administration (FDA) for the treatment of relapsed/refractory MCL. ${ }^{35}$ It is a reversible inhibitor of the ubiquitin-proteasome pathway (UPP), a complex pathway involving two distinct processes: 1) the poly-ubiquitination of intracellular proteins targeted for degradation, and 2) the $26 \mathrm{~S}$ proteasome-associated (the target for bortezomib) proteolysis. ${ }^{36}$ UPP plays a major role in the degradation of cellular proteins, and consequently affects a multitude of basic cellular processes. ${ }^{37}$ The precise mechanism of action of bortezomib in MCL is not entirely understood, but it likely involves the effect of UPP inhibition on several cellular pathways. These include direct impact on the cell-cycle regulation, proapoptotic/antiapoptotic balance proteins, and induction of endoplasmic reticulum (ER) stress and unfolded protein response. ${ }^{37}$

A hallmark in MCL is the overexpression of cyclin-D1, which plays a critical role in the cell-cycle progression. The level of the cyclin-dependent kinase inhibitor CDKN1B (p27 ${ }^{\text {Kip1 }}$ ) that can induce cell-cycle arrest by inhibiting cyclin-D1 is decreased in patients with MCL. ${ }^{38}$ The inhibition of the UPP by bortezomib leads to an increase in p $27^{\text {Kip } 1}$ level, and consequently a reduction in cyclin-D1 and regulation of the cell cycle. ${ }^{39}$

Proteasome inhibition by bortezomib has also been shown to upregulate the proapoptotic genes and downregulate antiapoptotic genes. ${ }^{40}$ The inhibition of the UPP decreases expression of the transcription factor, nuclear factor- $\kappa \mathrm{B}$, which is responsible for upregulation of genes that promote cell growth. ${ }^{41}$ Bortezomib also leads to an increase in the levels of NOXA, a proapoptotic protein. The induction of NOXA expression seems to be selective to the malignant cells only. ${ }^{35,42}$ Bortezomib has been shown to induce ER stress, ultimately leading to cytochrome c release, activation of caspases, and apoptosis..$^{35,43}$

Through their preclinical study on MCL cell lines and patient samples, Alinari et al showed that the combination of bortezomib and rituximab induced enhanced caspasedependent as well as independent apoptosis, decreased the levels of nuclear factor- $\kappa \mathrm{B}$ complexes, and diminished the activation of Akt pathway. ${ }^{44}$ Baiocchi et al demonstrated enhanced activity and improved survival with this combination in mice model. ${ }^{45}$ Bortezomib has also been shown to increase CD20 expression in rituximab-resistant cell lines and thus may play a role in reversing the acquired resistance to rituximab. ${ }^{46}$ Bortezomib in combination with rituximab and cyclophosphamide (BRC) leads to synergistic inhibition of growth in MCL cells and enhances apoptosis with early pan-caspase activation. In vivo studies showed that BRC eradicates subcutaneous tumors in MCL-bearing SCID mice and improves long-term event-free survival outcomes in $70 \%$ of the mice. ${ }^{47}$

The current recommended dose of bortezomib is $1.3 \mathrm{mg} / \mathrm{m}^{2}$ administered intravenously or subcutaneously on days 1, 4, 8, and 11 of a 21-day cycle. Subcutaneous administration has shown similar plasma concentration and pharmacodynamic profile compared with intravenous route at the same dose in patients with multiple myeloma. ${ }^{48}$ While no such studies have been conducted so far in MCL, subcutaneous route has the potential to reduce toxicity. Bortezomib requires no dose adjustments in patients with renal impairment, although moderate-to-severe hepatic impairment necessitates dose reduction to $0.7 \mathrm{mg} / \mathrm{m}^{2}{ }^{49,50}$ All patients receiving bortezomib require concurrent antiviral prophylaxis against herpes zoster.

\section{Efficacy of bortezomib and its comparison with other therapies in MCL}

The antitumor activity of bortezomib is evident in multiple studies in MCL, and this novel agent with its unique mechanism of action has been found to be effective in various clinical settings.

\section{Bortezomib for relapsed and/or refractory $\mathrm{MCL}$}

In an initial Phase II study involving patients with NHL during 2001-2003, 11 patients with relapsed/refractory MCL were administered bortezomib $\left(1.5 \mathrm{mg} / \mathrm{m}^{2}\right.$ on days $1,4,8$, and 11 in a 21-day cycle). Of the ten evaluable patients, one achieved a CR and four achieved PR (Table 3). ${ }^{51}$ 
Table 3 Phase II trials with bortezomib in relapsed/refractory $\mathrm{MCL}$

\begin{tabular}{|c|c|c|c|c|c|c|c|}
\hline $\begin{array}{l}\text { Study } \\
\text { type }\end{array}$ & Therapy & Dose of bortezomib ${ }^{a}$ & $\mathbf{N}$ & $\begin{array}{l}\text { Median OS } \\
\text { (months) }\end{array}$ & $\begin{array}{l}\text { Median PFS } \\
\text { (months) }\end{array}$ & $\begin{array}{l}\text { ORR } \\
(\%)\end{array}$ & $\begin{array}{l}\text { CR/CRu } \\
\text { (\%) }\end{array}$ \\
\hline Phase $\|^{29}$ & $\mathrm{~V}$ & $1.3 \mathrm{mg} / \mathrm{m}^{2}$; up to 17 cycles & 155 & 23.5 & 6.5 & 32 & 8 \\
\hline Phase II ${ }^{45}$ & $\checkmark$ with rituximab & $1.3-1.5 \mathrm{mg} / \mathrm{m}^{2} ; 5$ cycles & 14 & - & 2 & 29 & 29 \\
\hline Phase $\|^{51}$ & V & $1.5 \mathrm{mg} / \mathrm{m}^{2, b}$ & 10 & - & - & 50 & 10 \\
\hline Phase $\|^{52}$ & v & $1.5 \mathrm{mg} / \mathrm{m}^{2} ; 6$ cycles & 29 & - & - & 41 & 21 \\
\hline Phase $\|^{53}$ & $\vee$ & $1.3 \mathrm{mg} / \mathrm{m}^{2, c}$ & 15 & - & - & 47 & 7 \\
\hline Phase $I^{54}$ & $V$ with gemcitabine & $1.0 \mathrm{mg} / \mathrm{m}^{2} \mathrm{IV} \mathrm{c}$ & 25 & - & 11 & 60 & 12 \\
\hline Phase $I^{56}$ & $\begin{array}{l}V \text { with bendamustine } \\
\text { and rituximab }\end{array}$ & $1.3 \mathrm{mg} / \mathrm{m}^{2} ; 6$ cycles & 7 & - & - & 71 & - \\
\hline Phase $I^{55}$ & VRD & $1.3 \mathrm{mg} / \mathrm{m}^{2} ; 6$ cycles & 16 & 37 & 12 & 81 & 44 \\
\hline Phase $I^{75}$ & Temsirolimus and bortezomib & $\begin{array}{l}\text { Days } I, 8,15 \text {, and } 22 \text { of a } 35 \text { day cycle; } \\
\text { up to } 6 \text { cycles }\end{array}$ & 7 & - & 7.5 & 57 & 43 \\
\hline
\end{tabular}

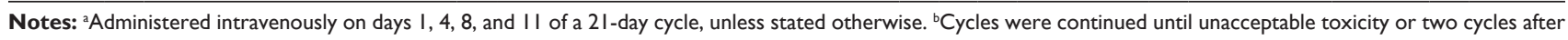
achieving CR. 'At least two cycles after CR or stable PR, four cycles after stable disease or until progressive disease.

Abbreviations: $C R$, complete response; CRu, complete response unconfirmed; D, dexamethasone; N, evaluable patients; ORR, overall response rate; OS, overall survival; PFS, progression-free survival; R, rituximab; V, bortezomib; VRD, bortezomib, rituximab, dexamethasone; $y$, years.

In another Phase II trial of 33 patients with relapsed/refractory MCL, an ORR of $41 \%(20.5 \% \mathrm{CR}, 20.5 \% \mathrm{PR})$ was noted (Table 3$).^{52}$

Bortezomib was ultimately approved in the USA in December 2006 for use in patients with relapsed or refractory MCL who had received at least one prior therapy, based on the results of the Phase II, PINNACLE study. ${ }^{29}$ In this study, 155 patients with relapsed/refractory MCL received single-agent bortezomib for up to 17 cycles or up to four cycles beyond achieving a $\mathrm{CR} /$ complete response unconfirmed $(\mathrm{CRu})$ or until progressive disease. An ORR of $32 \%$ with $8 \% \mathrm{CR} /$ $\mathrm{CRu}$ and a short median time-to-first response of 1.4 months were observed (Table 3). During the follow-up (median, 26.4 months), the median duration of response was 9.2 months and median TTP was 6.7 months (95\% CI: 5-10 months). Median OS was almost 2 years (95\% CI: 20-28 months). For responders, the median TTP was 12.4 months and the median OS was 35.4 months. Four deaths on study were considered to be related to bortezomib.

In the IND 150 trial conducted by Canadian Clinical Trials Group, 28 patients with MCL, 13 of whom were treatment-naive, were assessed for response to single-agent bortezomib. ${ }^{53}$ An ORR of $46 \%$ with one CRu and 12 PRs (44\%) were achieved. An association with bortezomib and fluid retention was noted. ${ }^{53}$ However, no such events were witnessed following a change in the study protocol to exclude patients with preexisting conditions predisposing to fluid retention.

Encouraging single-agent activity triggered interest in evaluating bortezomib-based combinations. The Canada Clinical Trials Group conducted IND 172 study examining the combination of bortezomib ( $1 \mathrm{mg} / \mathrm{m}^{2}$ IV on days $1,4,8$, and 11 of a 21 -day cycle) with gemcitabine $\left(1,000 \mathrm{mg} / \mathrm{m}^{2}\right.$ on days 1 and 8) in 25 patients with relapsed/refractory MCL who had received up to 1-3 prior therapies. ${ }^{54}$ An ORR of $60 \%$ with a median PFS of 11.4 months was observed. Although the PFS was better than that noted with single-agent bortezomib in the PINNACLE study (median PFS, 6.5 months), the patients enrolled in the PINNACLE study were more heavily pretreated. Most patients experienced hematological toxicities with bortezomib and gemcitabine combination. ${ }^{54}$ Pain and fatigue were common nonhematologic side effects. Of the three patients who experienced serious adverse events with this regimen, two had pleural effusions.

A Phase II trial of rituximab plus bortezomib (R-bortezomib) in the relapsed/refractory setting has shown an ORR of $29 \%$ with all responders achieving a CR by the third cycle. ${ }^{45}$ The median PFS was a dismal 1.9 months with $46 \%$ of the patients dying from disease progression (Table 3). Salvage R-bortezomib with additional dexamethasone showed a better ORR of $81 \%$ with a CR of $44 \%$ in another Phase II study. ${ }^{55}$ A promising activity was observed in this study, and patients achieving CR demonstrated sustained disease control (Table 3). Bortezomib has also been studied in combination with bendamustine and rituximab (Table 3) in a small Phase II study. ${ }^{56}$ A number of novel bortezomib-based combinations (discussed in the subsequent sections of our review) are being evaluated in MCL currently.

\section{Bortezomib in newly diagnosed $\mathrm{MCL}$}

An ORR of $46 \%$ attained with bortezomib monotherapy in MCL improves to $79 \%-95 \%$ with bortezomib-based combinations in the frontline setting, with CR rates improving to 53\%-64\% (Table 4) underscoring the value of bortezomib-chemotherapy combinations. The R-CHOP regimen has been commonly employed in newly diagnosed MCL, leading to a median 
Table 4 Phase II and III trials in newly diagnosed MCL

\begin{tabular}{|c|c|c|c|c|c|c|c|}
\hline Study type & Therapy & $\mathbf{N}$ & Median OS & Median PFS & ORR (\%) & CR/CRu (\%) & PR (\%) \\
\hline Phase IIIII (LYM-3002) & R-CHOP vs VR-CAP & 244 vs 243 & $56.3 \mathrm{~m}$ vs NR & $14.4 \mathrm{~m}$ vs $24.7 \mathrm{~m} *$ & 89 vs 92 & 42 vs $53^{*}$ & - \\
\hline Phase $\|^{53}$ & V & 13 & - & - & 46.2 & 0 & 46.2 \\
\hline Phase $1 I^{58}$ & VR-CHOP & 36 & $86 \%$ at $2 y$ & $23 \mathrm{~m}$ & 81 & 64 & - \\
\hline Phase II62 & VcR-CVAD with ASCT or MR & 30 & $86 \%$ at $3 y$ & $63 \%$ at $3 y$ & 90 & 70 & 13 \\
\hline Phase II ${ }^{64}$ & VcR-CVAD with MR & 75 & $88 \%$ at $3 y$ & $72 \%$ at $3 y$ & 95 & 68 & - \\
\hline Phase II65 & $\operatorname{RiPAD}+\mathrm{C}$ & 39 & NR & $26 \mathrm{~m}$ & 79 & 59 & \\
\hline Phase $11{ }^{66}(\mathrm{~S} 060 \mathrm{I})$ & $\begin{array}{l}\text { VR-CHOP followed by 2-year } \\
\text { V maintenance }\end{array}$ & 65 & $85 \%$ at $2 y$ & $62 \%$ at $2 y$ & - & - & - \\
\hline
\end{tabular}

Note: $* p<0.05$.

Abbreviations: CAP, cyclophosphamide, doxorubicin, prednisone; CHOP, cyclophosphamide, doxorubicin, vincristine, prednisone; CR, complete response; CRu, complete response unconfirmed; CVAD, cyclophosphamide, vincristine, doxorubicin, dexamethasone; m, months; MR, maintenance rituximab; N, evaluable patients; NR, not reached; ORR, overall response rate; OS, overall survival; PFS, progression-free survival; R, rituximab; RiPAD + C, rituximab, bortezomib, doxorubicin, dexamethasone, cyclophosphamide; V, bortezomib; VR or VcR-bortezomib, rituximab; y, years.

PFS of 16-20 months. ${ }^{14,57}$ Bortezomib was studied by Ruan et al in combination with the R-CHOP regimen in 36 patients of MCL ( $94 \%$ stage III or IV, and intermediate or high-risk MIPI in two-thirds) in a dose-escalation Phase I/II multicenter study (Table 4). ${ }^{58}$ The study suggested that bortezomib could be safely added to R-CHOP at doses up to $1.3 \mathrm{mg} / \mathrm{m}^{2}$ on days 1 and 4 of six 21-day cycles. A superior median PFS of 23 months was also observed with this combination in comparison to that seen with R-CHOP alone (16.6 months) and R-CHOP followed by either ASCT or interferon alpha maintenance (20 months) in two previously conducted trials. ${ }^{14,57}$

In LYM-3002 Phase III study involving 487 ASCTineligible newly diagnosed patients with MCL, R-CHOP was compared with VR-CAP, a regimen in which vincristine of R-CHOP was replaced by bortezomib. ${ }^{21}$ Bortezomib $\left(1.3 \mathrm{mg} / \mathrm{m}^{2}\right)$ was administered intravenously for six 3-week cycles, twice weekly (days 1, 4, 8, and 11) for the first 2 weeks followed by a 10 -day rest period in each cycle. Two additional cycles of VR-CAP were recommended for patients who first demonstrated a response at cycle 6 . The results of LYM-3002 led to the approval of VR-CAP regimen for frontline treatment of MCL in the USA, Europe, and Canada. Although there are several new targeted drugs approved by the FDA for patients with relapsed/refractory MCL, up to the year 2014, no therapy was approved for the treatment of patients with previously untreated MCL. VR-CAP resulted in a superior PFS (Table 4), irrespective of the baseline characteristics or Ki-67 expression status. However, the favorable impact on PFS was not appreciable in the MIPI high-risk category patients. The time-to-nexttreatment was 45 months with VR-CAP versus 24.8 months with R-CHOP, with the VR-CAP cohort demonstrating an advantage of a median period of 1.7 years without therapy. No survival advantage was discernible at last follow-up. The 4-year OS rate was 64\% with VR-CAP versus 54\% with R-CHOP, and longer follow-up is required to assess the true OS benefit with VR-CAP. Although the ORR was similar between the two regimens, the depth of response was significantly better with VR-CAP (CR 53\% vs $42 \%$ with R-CHOP; $P=0.0007)$. The rate of discontinuation of therapy due to adverse events was similar between the two regimens. However, grade 3 or higher adverse events were more common with VR-CAP (93\% vs $85 \%$ with R-CHOP). Hematologic toxicity was the most common adverse effect observed in both the groups, with thrombocytopenia being much more frequent with VR-CAP (72\% vs 19\% with R-CHOP). Grade 3 or higher adverse events of neutropenia and infections were observed in $85 \%$ and $21 \%$ of the patients, respectively, in the VR-CAP arm. The rates of peripheral neuropathy $(\mathrm{PN})$ were similar in the two groups as one neurotoxic agent (vincristine) was replaced with the other (bortezomib) between the two groups, but fortunately, $\mathrm{PN}$ was reversible in the majority of the patients. The rate of neurotoxicity with bortezomib (30\%) was lower compared with that typically observed in the patients with myeloma $(37 \%-44 \%) .{ }^{59-61}$ VR-CAP was associated with the discontinuation rate of $8 \% .{ }^{21}$ Serious adverse events like febrile neutropenia and pneumonia were seen in $11 \%$ and $8 \%$ of the patients, respectively.

Importantly, neither did the LYM-3002 study utilize subcutaneous route of administration of bortezomib, which is associated with reduced toxicity, nor did it explore the role of rituximab or bortezomib maintenance.

The ongoing Intergroup study randomizing the patients $\geq 60$ years of age with previously untreated MCL to BR versus BR plus bortezomib arms followed by a second randomization of rituximab versus lenalidomide plus rituximab maintenance (Table 5) would help shed more light on the impact of maintenance strategies following bortezomibbased therapy.

In an attempt to build upon their previous experience with the less-intensive regimen of modified R-hyperCVAD 
Table 5 Ongoing trials of bortezomib in mantle cell lymphoma

\begin{tabular}{llll}
\hline Phase & Identifier & Patient population & Therapy \\
\hline I & NCT0067III2 & R/R & Everolimus and bortezomib \\
I & NCT0IIIII I88 & R/R & Palbociclib plus bortezomib \\
I & NCT0I69594I & R/R & Alisertib, bortezomib, and rituximab \\
I/II & NCT02356458 & R/R & lbrutinib and bortezomib \\
I/II & NCT0I439750 & TN and R/R & Bortezomib, cladribine, and rituximab \\
I/II & NCT0I322776 & R/R & Bortezomib, fludarabine, and cyclophosphamide \\
II & NCT00II4738 & TN & EPOCH-R \pm bortezomib \\
II & NCT0I2678I2 & Post ASCT & Weekly maintenance bortezomib and rituximab \\
II & NCT0I4I5752 & TN & Rituximab, bendamustine, and bortezomib followed by rituximab and lenalidomide \\
II & NCT00992446 & Post ASCT & Bortezomib and vorinostat as maintenance therapy \\
II & NCT0I457I44 & TN & Rituximab, bortezomib, bendamustine, and dexamethasone (RiBVD) \\
III & NCT0I449344 & R/R & Rituximab, cytarabine, dexamethasone, and bortezomib \\
\hline
\end{tabular}

Abbreviations: ASCT, autologous stem cell transplant; EPOCH-R, prednisone, etoposide, doxorubicin, vincristine, and rituximab; R/R, relapsed/refractory; TN, treatment naive.

chemotherapy with MR resulting in 3-year PFS of 50\%, the Wisconsin Oncology Network Study used bortezomib (VcRCVAD) along with extended MR (beyond 2 years). Designed with the intent of improving the CR rate by the addition of bortezomib and the durability of remission through extended rituximab maintenance, the trial studied 30 newly diagnosed patients with MCL who received VcR-CVAD chemotherapy every 21 days for six cycles. Patients achieving at least a PR went on to receive rituximab consolidation $\left(375 \mathrm{mg} / \mathrm{m}^{2}\right.$ for 4 weekly doses) followed by MR $\left(375 \mathrm{mg} / \mathrm{m}^{2}\right.$ every 12 weeks for 20 doses). All patients had advanced-stage disease and $60 \%$ had a medium-to-high MIPI score. ${ }^{62}$ The response rates achieved with VcR-CVAD (Table 4) were better than those seen historically with the modified R-hyper-CVAD. Although 3-year PFS and OS were comparably better than the outcomes with modified R-hyper-CVAD regimen alone, a more intensive regimen of 21 days instead of 28 days was used in this study ${ }^{63}$ Myelosuppression of comparable severity was the major toxicity with both regimens. ${ }^{62,63} \mathrm{PN}$ was more common with VcR-CVAD but adequately managed with necessary dose reductions in bortezomib and vincristine.

The Eastern Cooperative Oncology Group (ECOG) conducted a similar but a larger Phase II multicenter study (E1405) with VcR-CVAD involving 75 treatment naive patients with MCL with a goal of assessing the impact of additional bortezomib. ${ }^{64}$ Transplant-eligible patients were additionally given an option of receiving ASCT consolidation instead of rituximab maintenance post-induction (Table 4). The CR rate was a conservative estimate since some patients were coded as PR as a result of missing end-of-treatment evaluations. No difference was observed in the outcomes of patients who underwent ASCT versus those who received rituximab maintenance. Myelosuppression was the most common toxicity with VcR-CVAD regimen. Grade 3 or higher PN was not evident with the use of modified doses of bortezomib (1.3 mg/m² IV on days 1 and 4$)$ and vincristine ( $1 \mathrm{mg}$ IV on day 3 per cycle). The induction regimen was also associated with high adherence with nearly $90 \%$ of patients completing all six cycles. None of the patients experienced grade 3/4 PN in the E1405 trial in which the reduced dosage was adopted from the beginning. The results of this trial confirmed the efficacy of incorporation of bortezomib, providing foundation for the currently ongoing E1411 trial (NCT01415752; Table 5) investigating the role of addition of bortezomib to BR.

The Groupe Ouest Est d'Etude des Leucémies aiguës et Autres Maladies du Sang (GOELAMS) had developed RVAD + C (rituximab, vincristine, doxorubicin, dexamethasone, and chlorambucil), a frontline regimen for elderly patients that was found to be as effective as R-CHOP, but less toxic. The group subsequently conducted a Phase II study replacing vincristine with bortezomib in the combination (RiPAD $+\mathrm{C}$ ) to enhance its efficacy in elderly patients. ${ }^{65}$ Thirty-nine patients with a median age of 72 years (range: 65-80) were enrolled in this study (Table 4), and the outcomes were superior compared with those seen with $\mathrm{RVAD}+\mathrm{C}$, despite a greater proportion of patients treated with RiPAD $+\mathrm{C}$ having an aggressive disease (30\% vs $18 \%$ blastoid subtypes). The response rates (CR rate $60 \%$ vs 34-48\%) and survival outcomes (median PFS, 26 months vs 16-20 months) were also better in comparison with those seen with R-CHOP in the studies involving younger patients and better prognostic MIPI scores. ${ }^{14,57}$ The discontinuation rate was only $10 \%$ (4/39) with this regimen. However, myelosuppression and neurotoxicity were common with a significantly higher proportion of patients requiring transfusions 
(24\%) and/or developing neurotoxicity (45\%). Nearly $18 \%$ experienced grade 3 or higher neuropathy with intravenous bortezomib in this trial, an issue which could probably be alleviated without compromising the regimen's efficacy by switching to the subcutaneous route of administration. As frontline therapy, RiPAD $+\mathrm{C}$ regimen has been associated with a high hospitalization rate of 34\% (median duration of 7 days). ${ }^{65}$ A total of $10 \%$ of the patients had to discontinue treatment prematurely due to toxicity. Two out of 39 patients in the study died due to severe sepsis.

\section{Bortezomib consolidation or maintenance in $\mathrm{MCL}$}

The South West Oncology Group (SWOG) investigated the impact of adding bortezomib to R-CHOP for induction (at a schedule previously established by Ruan et al) followed by bortezomib maintenance $\left(1.3 \mathrm{mg} / \mathrm{m}^{2}\right.$ days $1,4,8$, and 11 of every 3-month cycle for eight cycles) for 2 years. ${ }^{58,66}$ The preliminary results indicated that 65 eligible patients were treated, and that the estimated 2-year PFS and OS were $62 \%$ and $85 \%$, respectively, suggesting near doubling of the historical 2-year PFS with R-CHOP alone. ${ }^{14,57}$ Hematological toxicities of grade 4 were experienced by $48 \%$ of patients. Surprisingly, despite concomitant use of vincristine and bortezomib, the investigators reported grade $3 \mathrm{PN}$ in only $8 \%$ of patients during induction and $2 \%$ during maintenance phase. Owing to its design of utilizing bortezomib in both the induction and the maintenance phases, the independent impact of the addition of bortezomib at each phase on the outcome could not be ascertained from this study.

The Cancer and Leukemia Group B (CALGB) developed an intensive regimen, CALGB 59909 involving highdose chemotherapy followed by ASCT with rituximab in untreated patients with MCL. ${ }^{67}$ It was associated with an impressive 5 -year OS of $64 \%$. In the subsequent CALGB 50403 trial, the patients were randomized after the administration of the CALGB 59909 regimen to receive bortezomib either as consolidation (BC; $1.3 \mathrm{mg} / \mathrm{m}^{2}$ on days $1,4,8$, and 11 for up to four 3-week cycles) or as maintenance (BM; $1.6 \mathrm{mg} / \mathrm{m}^{2}$ on days $1,8,15$, and 22 for up to ten 8 -week cycles).$^{68}$ Preliminary analysis revealed that the 2 -year PFS were $89 \%$ and $84 \%$ post-randomization with $\mathrm{BC}$ and BM, respectively. In the patients who underwent ASCT, the posttransplant 3-year PFS was significantly better in the CALGB 50403 study $(n=118)$ compared with the CALGB 59909 study ( $\mathrm{n}=66 ; 3$-year $\mathrm{PFS}, 67 \%$ vs $59 \% ; P=0.0086$ ). The completion rates with both consolidation and maintenance bortezomib regimens were over $65 \%$, although consolidation therapy was associated with slightly higher rates of adverse events.

\section{Toxicities}

As discussed earlier, with the passage of time, clinicians have gained substantial experience in using bortezomib-based regimens and managing their toxicities more effectively. Bortezomib monotherapy has arguably a lower rate of toxicity with grade $\geq 3$ neutropenia seen only in $7 \%$ and grade $\geq 3$ PN in only $8 \%$ of the patients. ${ }^{53} \mathrm{PN}$ appears to resolve in the majority of the patients $(80 \%)$ within a few months of reduction or discontinuation of bortezomib. Fatigue (72\%), diarrhea (48\%), and anorexia (35\%) are other common nonhematologic side effects. The incidence of pyrexia with bortezomib is higher $(23 \%)$ among patients with myeloma compared with those with MCL $(10 \%)$ while rates of orthostatic hypotension are similar (8\%-9\%). Maintenance therapy with bortezomib has been associated with grade 3 nonhematologic toxicities in $13 \%$ and grade $3 \mathrm{PN}$ in only $2 \%$ of patients.

Although no published studies in MCL have specifically reported on the impact on QoL outcomes with the addition of bortezomib, neurotoxicity is a major concern that has the potential to adversely affect patients' QoL.

\section{Future directions}

Buoyed by its recent approval in the USA and the European Union in the frontline setting in combination with rituximab, cyclophosphamide, doxorubicin, and prednisone, the role of bortezomib in MCL is poised to expand in the coming years. Indeed a wide array of trials using other combination regimens integrated with bortezomib backbone is underway. Bortezomib is currently being investigated in combination with newer agents including everolimus (an mTOR inhibitor), alisertib (an aurora A kinase inhibitor), and palbociclib (a CDK 4 and 6 inhibitor; Table 5). It is also being studied in a Phase I/II trial in combination with ibrutinib. ${ }^{27}$ The highly effective combination of bendamustine and rituximab is also being studied in multiple Phase II trials in combination with bortezomib as frontline therapy in patients with MCL. Bortezomib in combination with rituximab, high-dose cytarabine, and dexamethasone is another regimen currently under evaluation in a Phase III trial in relapsed or refractory MCL. Its role as maintenance therapy post-ASCT is also being investigated further (Table 5). The future of this proteasome inhibitor appears to be exciting and promising in MCL as researchers continue to explore its efficacy through a multitude of studies in various clinical settings. 


\section{Disclosure}

PK reports receiving research funding from Takeda and Onyx Pharmaceuticals (an Amgen subsidiary). The authors report no other conflicts of interest in this work.

\section{References}

1. Dreyling M, Geisler C, Hermine O, et al; ESMO Guidelines Working Group. Newly diagnosed and relapsed mantle cell lymphoma: ESMO clinical practice guidelines for diagnosis, treatment and follow-up. Ann Oncol. 2014;25(suppl 3):iii83-iii92.

2. Smith A, Crouch S, Lax S, et al. Lymphoma incidence, survival and prevalence 2004-2014: sub-type analyses from the UK's Haematological Malignancy Research Network. Br J Cancer. 2015;112(9): $1575-1584$.

3. Wang Y, Ma S. Racial differences in mantle cell lymphoma in the United States. BMC Cancer. 2014;14:764.

4. Argatoff LH, Connors JM, Klasa RJ, Horsman DE, Gascoyne RD. Mantle cell lymphoma: a clinicopathologic study of 80 cases. Blood. 1997; 89(6):2067-2078.

5. Martin P, Chadburn A, Christos $P$, et al. Outcome of deferred initial therapy in mantle-cell lymphoma. J Clin Oncol. 2009;27(8):1209-1213.

6. Fernàndez V, Salamero $\mathrm{O}$, Espinet $\mathrm{B}$, et al. Genomic and gene expression profiling defines indolent forms of mantle cell lymphoma. Cancer Res. 2010;70(4):1408-1418.

7. Navarro A, Clot G, Royo C, et al. Molecular subsets of mantle cell lymphoma defined by the IGHV mutational status and SOX11 expression have distinct biologic and clinical features. Cancer Res. 2012;72(20):5307-5316.

8. Martin P, Leonard J. Is there a role for "watch and wait" in patients with mantle cell lymphoma? Semin Hematol. 2011;48(3):189-193.

9. Hoster E, Dreyling M, Klapper W, et al; German Low Grade Lymphoma Study Group (GLSG), European Mantle Cell Lymphoma Network. A new prognostic index (MIPI) for patients with advanced-stage mantle cell lymphoma. Blood. 2008;111(2):558-565.

10. Schulz H, Bohlius JF, Trelle S, et al. Immunochemotherapy with rituximab and overall survival in patients with indolent or mantle cell lymphoma: a systematic review and meta-analysis. $J$ Natl Cancer Inst. 2007;99(9):706-714.

11. Griffiths R, Mikhael J, Gleeson M, Danese M, Dreyling M. Addition of rituximab to chemotherapy alone as first-line therapy improves overall survival in elderly patients with mantle cell lymphoma. Blood. 2011;118(18):4808-4816

12. Hoster E, Metzner B, Forstpointner R, et al. Autologous stem cell transplantation and addition of rituximab independently prolong response duration in advanced stage mantle cell lymphoma. ASH Аnпи Meet Abstr. 2009;114(22):880.

13. Kluin-Nelemans HC, Hoster E, Hermine O, et al. Treatment of older patients with mantle-cell lymphoma. N Engl J Med. 2012;367(6):520-531.

14. Lenz G, Dreyling M, Hoster E, et al. Immunochemotherapy with rituximab and cyclophosphamide, doxorubicin, vincristine, and prednisone significantly improves response and time to treatment failure, but not longterm outcome in patients with previously untreated mantle cell lymphoma: results of a prospective randomized trial of the German Low Grade Lymphoma Study Group (GLSG). J Clin Oncol. 2005;23(9):1984-1992.

15. Hermine O, Hoster E, Walewski J, et al. Alternating courses of $3 x$ CHOP and 3x DHAP plus rituximab followed by a high dose ARA-C containing myeloablative regimen and autologous stem cell transplantation (ASCT) increases overall survival when compared to 6 courses of CHOP plus rituximab followed by myeloablative radiochemotherapy and ASCT in mantle cell lymphoma: final analysis of the MCL younger trial of the European Mantle Cell Lymphoma Network (MCL net). ASH Annu Meet Abstr. 2012;120(21):151.

16. Romaguera JE, Fayad LE, Feng L, et al. Ten-year follow-up after intense chemoimmunotherapy with rituximab-HyperCVAD alternating with rituximab-high dose methotrexate/cytarabine (R-MA) and without stem cell transplantation in patients with untreated aggressive mantle cell lymphoma. Br J Haematol. 2010;150(2):200-208.
17. Merli F, Luminari S, Ilariucci F, et al. Rituximab plus HyperCVAD alternating with high dose cytarabine and methotrexate for the initial treatment of patients with mantle cell lymphoma, a multicentre trial from Gruppo Italiano Studio Linfomi. Br J Haematol. 2012;156(3): 346-353.

18. Bernstein SH, Epner E, Unger JM, et al. A phase II multicenter trial of hyperCVAD MTX/Ara-C and rituximab in patients with previously untreated mantle cell lymphoma; SWOG 0213. Ann Oncol. 2013; 24(6):1587-1593.

19. Rummel MJ, Niederle N, Maschmeyer G, et al; Study Group Indolent Lymphomas (STIL). Bendamustine plus rituximab versus CHOP plus rituximab as first-line treatment for patients with indolent and mantlecell lymphomas: an open-label, multicentre, randomised, phase 3 non-inferiority trial. Lancet. 2013;381(9873):1203-1210.

20. Flinn IW, van der Jagt R, Kahl BS, et al. Randomized trial of bendamustine-rituximab or R-CHOP/R-CVP in first-line treatment of indolent NHL or MCL: the BRIGHT study. Blood. 2014;123(19):2944-2952.

21. Robak T, Huang H, Jin J, et al; LYM-3002 Investigators. Bortezomibbased therapy for newly diagnosed mantle-cell lymphoma. $N$ Engl $J$ Med. 2015;372(10):944-953.

22. Bauwens D, Maerevoet M, Michaux L, et al. Activity and safety of combined rituximab with chlorambucil in patients with mantle cell lymphoma. Br J Haematol. 2005;131(3):338-340.

23. Sachanas S, Pangalis GA, Vassilakopoulos TP, et al. Combination of rituximab with chlorambucil as first line treatment in patients with mantle cell lymphoma: a highly effective regimen. Leuk Lymphoma. 2011;52(3):387-393.

24. Coleman M, Martin P, Ruan J, et al. Low-dose metronomic, multidrug therapy with the PEP-C oral combination chemotherapy regimen for mantle cell lymphoma. Leuk Lymphoma. 2008;49(3):447-450.

25. Morschhauser FA, Cartron G, Thieblemont C, et al. Obinutuzumab (GA101) monotherapy in relapsed/refractory diffuse large b-cell lymphoma or mantle-cell lymphoma: results from the phase II GAUGUIN study. J Clin Oncol. 2013;31(23):2912-2919.

26. Furtado M, Dyer MJ, Johnson R, Berrow M, Rule S. Ofatumumab monotherapy in relapsed/refractory mantle cell lymphoma - a phase II trial. Br J Haematol. 2014;165(4):575-578.

27. Wang ML, Rule S, Martin P, et al. Targeting BTK with ibrutinib in relapsed or refractory mantle-cell lymphoma. N Engl J Med. 2013; 369(6):507-516.

28. Maddocks K, Christian B, Jaglowski S, et al. A phase 1/1b study of rituximab, bendamustine, and ibrutinib in patients with untreated and relapsed/refractory non-Hodgkin lymphoma. Blood. 2015; 125(2):242-248.

29. Goy A, Bernstein SH, Kahl BS, et al. Bortezomib in patients with relapsed or refractory mantle cell lymphoma: updated time-to-event analyses of the multicenter phase 2 PINNACLE study. Ann Oncol. 2009;20(3):520-525.

30. Goy A, Sinha R, Williams ME, et al. Single-agent lenalidomide in patients with mantle-cell lymphoma who relapsed or progressed after or were refractory to bortezomib: phase II MCL-001 (EMERGE) study. J Clin Oncol. 2013;31(29):3688-3695.

31. Hess G, Herbrecht R, Romaguera J, et al. Phase III study to evaluate temsirolimus compared with investigator's choice therapy for the treatment of relapsed or refractory mantle cell lymphoma. J Clin Oncol. 2009;27(23):3822-3829.

32. Trneny M, Lamy T, Walewski JA, et al. Subgroup analysis of the phase II randomized MCL-002 (SPRINT) study of lenalidomide vs investigator's choice in relapsed/refractory mantle cell lymphoma. ASCO Meet Abstr. 2015;33(15 suppl):8547.

33. Wang Y, Dela Rosa ML, Zhou S, et al. Lenalidome in combination with rituximab for relapsed or refractory mantel cell lymphoma: updated analysis of a phase 2 trial. ASCO Meet Abstr. 2015;33(15 suppl):8542.

34. Kahl B, Byrd JC, Flinn IW, et al. Clinical safety and activity in a phase 1 study of CAL-101, an isoform-selective inhibitor of phosphatidylinositol 3-kinase $\mathrm{P} 110\{$ delta\}, in patients with relapsed or refractory non-Hodgkin lymphoma. ASH Annu Meet Abstr. 2010; 116(21):1777. 
35. Mujtaba T, Dou QP. Advances in the understanding of mechanisms and therapeutic use of bortezomib. Discov Med. 2011;12(67):471-480.

36. Spataro V, Norbury C, Harris AL. The ubiquitin-proteasome pathway in cancer. Br J Cancer. 1998;77(3):448-455.

37. Adams J. The proteasome: structure, function, and role in the cell. Cancer Treat Rev. 2003;29(suppl 1):3-9.

38. Pagano M, Tam SW, Theodoras AM, et al. Role of the ubiquitinproteasome pathway in regulating abundance of the cyclin-dependent kinase inhibitor p27. Science. 1995;269(5224):682-685.

39. Paoluzzi L, O'Connor OA. Mechanistic rationale and clinical evidence for the efficacy of proteasome inhibitors against indolent and mantle cell lymphomas. BioDrugs. 2006;20(1):13-23.

40. Mitsiades N, Mitsiades CS, Poulaki V, et al. Molecular sequelae of proteasome inhibition in human multiple myeloma cells. Proc Natl Acad Sci U S A. 2002;99(22):14374-14379.

41. Palombella VJ, Rando OJ, Goldberg AL, Maniatis T. The ubiquitinproteasome pathway is required for processing the NF-kappa B1 precursor protein and the activation of NF-kappa B. Cell. 1994;78(5): 773-785.

42. Qin JZ, Ziffra J, Stennett L, et al. Proteasome inhibitors trigger NOXAmediated apoptosis in melanoma and myeloma cells. Cancer Res. 2005; 65(14):6282-6293

43. Landowski TH, Megli CJ, Nullmeyer KD, Lynch RM, Dorr RT Mitochondrial-mediated disregulation of $\mathrm{Ca} 2+$ is a critical determinant of Velcade (PS-341/bortezomib) cytotoxicity in myeloma cell lines. Cancer Res. 2005;65(9):3828-3836.

44. Alinari L, White VL, Earl CT, et al. Combination bortezomib and rituximab treatment affects multiple survival and death pathways to promote apoptosis in mantle cell lymphoma. MAbs. 2009;1(1):31-40.

45. Baiocchi RA, Alinari L, Lustberg ME, et al. Phase 2 trial of rituximab and bortezomib in patients with relapsed or refractory mantle cell and follicular lymphoma. Cancer. 2011;117(11):2442-2451.

46. Czuczman MS, Olejniczak S, Gowda A, et al. Acquirement of rituximab resistance in lymphoma cell lines is associated with both global CD20 gene and protein down-regulation regulated at the pretranscriptional and posttranscriptional levels. Clin Cancer Res. 2008;14(5): 1561-1570.

47. Wang M, Han XH, Zhang L, et al. Bortezomib is synergistic with rituximab and cyclophosphamide in inducing apoptosis of mantle cell lymphoma cells in vitro and in vivo. Leukemia. 2008;22(1):179-185.

48. Moreau P, Karamanesht II, Domnikova N, et al. Pharmacokinetic, pharmacodynamic and covariate analysis of subcutaneous versus intravenous administration of bortezomib in patients with relapsed multiple myeloma. Clin Pharmacokinet. 2012;51(12):823-829.

49. Leal TB, Remick SC, Takimoto $\mathrm{CH}$, et al. Dose-escalating and pharmacological study of bortezomib in adult cancer patients with impaired renal function: a national cancer institute organ dysfunction working group study. Cancer Chemother Pharmacol. 2011;68(6): 1439-1447.

50. LoRusso PM, Venkatakrishnan K, Ramanathan RK, et al. Pharmacokinetics and safety of bortezomib in patients with advanced malignancies and varying degrees of liver dysfunction: phase I NCI organ dysfunction working group study NCI-6432. Clin Cancer Res. 2012;18(10):2954-2963.

51. O'Connor OA, Wright J, Moskowitz C, et al. Phase II clinical experience with the novel proteasome inhibitor bortezomib in patients with indolent non-Hodgkin's lymphoma and mantle cell lymphoma. J Clin Oncol. 2005;23(4):676-684.

52. Goy A, Younes A, McLaughlin P, et al. Phase II study of proteasome inhibitor bortezomib in relapsed or refractory B-cell non-Hodgkin's lymphoma. J Clin Oncol. 2005;23(4):667-675.

53. Belch A, Kouroukis CT, Crump M, et al. A phase II study of bortezomib in mantle cell lymphoma: the national cancer institute of Canada clinical trials group trial IND.150. Ann Oncol. 2007;18(1):116-121.

54. Kouroukis CT, Fernandez LA, Crump M, et al. A phase II study of bortezomib and gemcitabine in relapsed mantle cell lymphoma from the national cancer institute of Canada clinical trials group (IND 172). Leuk Lymphoma. 2011;52(3):394-399.
55. Lamm W, Kaufmann H, Raderer M, et al. Bortezomib combined with rituximab and dexamethasone is an active regimen for patients with relapsed and chemotherapy-refractory mantle cell lymphoma. Haematologica. 2011;96(7):1008-1014.

56. Friedberg JW, Vose JM, Kelly JL, et al. The combination of bendamustine, bortezomib, and rituximab for patients with relapsed/refractory indolent and mantle cell non-Hodgkin lymphoma. Blood. 2011;117(10): 2807-2812.

57. Howard OM, Gribben JG, Neuberg DS, et al. Rituximab and CHOP induction therapy for newly diagnosed mantle-cell lymphoma: molecular complete responses are not predictive of progression-free survival. J Clin Oncol. 2002;20(5):1288-1294.

58. Ruan J, Martin P, Furman RR, et al. Bortezomib plus CHOP-rituximab for previously untreated diffuse large B-cell lymphoma and mantle cell lymphoma. J Clin Oncol. 2011;29(6):690-697.

59. Richardson PG, Barlogie B, Berenson J, et al. A phase 2 study of bortezomib in relapsed, refractory myeloma. $N$ Engl J Med. 2003; 348(26):2609-2617.

60. Jagannath S, Barlogie B, Berenson J, et al. A phase 2 study of two doses of bortezomib in relapsed or refractory myeloma. Br J Haematol. 2004; 127(2):165-172.

61. Mateos MV, Richardson PG, Schlag R, et al. Bortezomib plus melphalan and prednisone compared with melphalan and prednisone in previously untreated multiple myeloma: updated follow-up and impact of subsequent therapy in the phase III VISTA trial. J Clin Oncol. 2010;28(13): 2259-2266.

62. Chang JE, Peterson C, Choi S, et al. VcR-CVAD induction chemotherapy followed by maintenance rituximab in mantle cell lymphoma: a Wisconsin oncology network study. Br J Haematol. 2011;155(2): 190-197.

63. Kahl BS, Longo WL, Eickhoff JC, et al; Wisconsin Oncology Network. Maintenance rituximab following induction chemoimmunotherapy may prolong progression-free survival in mantle cell lymphoma: a pilot study from the Wisconsin oncology network. Ann Oncol. 2006;17(9):1418-1423.

64. Chang JE, Li H, Smith MR, et al. Phase 2 study of VcR-CVAD with maintenance rituximab for untreated mantle cell lymphoma: an Eastern cooperative oncology group study (E1405). Blood. 2014;123(11): $1665-1673$

65. Houot R, Le Gouill S, Ojeda Uribe M, et al; French GOELAMS Group. Combination of rituximab, bortezomib, doxorubicin, dexamethasone and chlorambucil (RiPAD+C) as first-line therapy for elderly mantle cell lymphoma patients: results of a phase II trial from the GOELAMS. Ann Oncol. 2012;23(6):1555-1561.

66. Till BG, Li H, Bernstein SH, et al. Phase II trial of R-CHOP plus bortezomib induction therapy followed by bortezomib maintenance for previously untreated mantle cell lymphoma: SWOG 0601. ASH Annu Meet. 2014;124(21):149.

67. Damon LE, Johnson JL, Niedzwiecki D, et al. Immunochemotherapy and autologous stem-cell transplantation for untreated patients with mantle-cell lymphoma: CALGB 59909. J Clin Oncol. 2009;27(36): 6101-6108.

68. Kaplan L, Jung S, Bartelet N, et al. Bortezomib maintenance (BM) versus consolidation $(\mathrm{BC})$ following aggressive immunochemotherapy and autologous stem cell transplant (ASCT) for untreated mantle cell lymphoma (MCL): CALGB 50403. Hematol Oncol. 2013;31(S1):126.

69. Dreyling M, Lenz G, Hoster E, et al. Early consolidation by myeloablative radiochemotherapy followed by autologous stem cell transplantation in first remission significantly prolongs progression-free survival in mantle-cell lymphoma: results of a prospective randomized trial of the European MCL Network. Blood. 2005;105(7):2677-2684.

70. van 't Veer MB, de Jong D, MacKenzie M, et al. High-dose Ara-C and beam with autograft rescue in R-CHOP responsive mantle cell lymphoma patients. Br J Haematol. 2009;144(4):524-530.

71. Geisler CH, Kolstad A, Laurell A, et al; Nordic Lymphoma Group. Long-term progression-free survival of mantle cell lymphoma after intensive front-line immunochemotherapy with in vivo-purged stem cell rescue: a nonrandomized phase 2 multicenter study by the Nordic Lymphoma Group. Blood. 2008;112(7):2687-2693. 
72. Delarue R, Haioun C, Ribrag V, et al; Groupe d'Etude des Lymphomes de l'Adulte (GELA). CHOP and DHAP plus rituximab followed by autologous stem cell transplantation in mantle cell lymphoma: a phase 2 study from the Groupe d'Etude des Lymphomes de l'Adulte. Blood. 2013;121(1):48-53.

73. Romaguera JE, Fayad L, Rodriguez MA, et al. High rate of durable remissions after treatment of newly diagnosed aggressive mantlecell lymphoma with rituximab plus hyper-CVAD alternating with rituximab plus high-dose methotrexate and cytarabine. J Clin Oncol. 2005;23(28):7013-7023.
74. Inwards DJ, Fishkin PA, Hillman DW, et al. Long-term results of the treatment of patients with mantle cell lymphoma with cladribine (2-CDA) alone (95-80-53) or 2-CDA and rituximab (N0189) in the North central cancer treatment group. Cancer. 2008;113(1):108-116.

75. Fenske TS, Shah NM, Kim KM, et al. A phase 2 study of weekly temsirolimus and bortezomib for relapsed or refractory B-cell non-Hodgkin lymphoma: a Wisconsin oncology network study. Cancer. 2015; 121(19):3465-3471.

\section{Publish your work in this journal}

Therapeutics and Clinical Risk Management is an international, peerreviewed journal of clinical therapeutics and risk management, focusing on concise rapid reporting of clinical studies in all therapeutic areas, outcomes, safety, and programs for the effective, safe, and sustained use of medicines. This journal is indexed on PubMed Central, CAS,
EMBase, Scopus and the Elsevier Bibliographic databases. The manuscript management system is completely online and includes a very quick and fair peer-review system, which is all easy to use. Visit http://www.dovepress.com/testimonials.php to read real quotes from published authors.

Submit your manuscript here: http://www.dovepress.com/therapeutics-and-clinical-risk-management-journal 